care; 2) To increase asthma screening from $0 \%$ to $80 \%$ by December 2020.

Methods We developed an algorithm for asthma screening in EHR based on national guidelines that asked about medications taken at home, exercise-induced symptoms, medication adherence, daytime and night-time symptoms, use of rescue medication and interference with activity in the past 4 weeks, and use of oral corticosteroids in the past year. Screening was conducted by the nursing staff prior to patient being seen by the provider. Based on responses to the screening questions, severity/control classification is calculated and displayed in EHR for provider to then treat patient accordingly. Classic Quality Improvement tools such as the Model for Improvement and Plan Do Study Act (PDSA) cycles were used to incorporate changes into daily workflow. Practice Advisory Board met monthly to discuss barriers to implementation and identify solutions. The Board made decisions on adopting successful strategies and brainstormed new strategies to address barriers.

Results This project is ongoing and preliminary results are available from 3 practices: small pediatric, large pediatric and family medicine practices. The ultimate goal is to improve care and clinical outcomes of children with asthma. To date, a total of 4,057 screenings have been completed at these sites. Screening rates improved from $0 \%$ to $78.6 \%$ in a small pediatric practice, from $0 \%$ to $66.7 \%$ in a large pediatric practice, and from $0 \%$ to $38.7 \%$ in a family practice. Several problems were encountered during the process of change: competition with other nurse-completed screenings; screening questions are available in English only within EHR; nursing staff shortages; nurses forgetting to conduct screening; and caregiver not knowing information about child's asthma or disagreeing with asthma diagnosis.

Conclusion We developed an asthma screening algorithm within EHR, trained nursing staff and implemented patient screening at the time of clinic visit allowing providers take action on asthma management based on the screening result. Further collaborative efforts are needed to improve and sustain screening rates with the ultimate goal of improving patient clinical outcomes.

\section{GP155 ARE CLINICAL PREDICTION RULES IN PAEDIATRICS VALIDATED? A LITERATURE REVIEW}

CA Keegan Hickey*, ZA Sarani, MB O'Neill. Mayo University Hospital, Castlebar, Ireland

10.1136/archdischild-2019-epa.219

Aims To identify and appraise the geographical and temporal transportability of externally validated clinical prediction rules (CPRs) in the paediatric population.

Methods A Medline search was conducted from 2000-2015 for publications involving the derivation, internal or external validation of a CPR in children aged $0-21$ years. Validation studies were included if they described a CPR which was developed within this time frame.

Results 193 studies were identified for inclusion. The selected studies describe 133 CPRs, across more than 10 paediatric specialities. 63 CPRs were not validated (47\%). Of the 70 validated rules identified (53\%), 25 were internally validated, 8 were cross validated and 53 were externally validated. Only 13 CPRs (10\%) were both internally and externally validated and 21 CPRs (19\%) were externally validated more than once. 15 internally validated studies used split sample methodology while only 5 performed bootstrapping regression methods. Of the 87 studies measuring external validity, the majority of studies (66\%) were performed at a different site to the derivation. Only 5 studies were performed at the same site while 22 did not disclose this information. 43 externally validated studies included populations that were recruited in a different country to the derivation and 27 of those were on a different continent. Only two externally validated studies were shown not to have temporal transportability. Performance of the rules in validation was poorly reported, and if reported usually underperformed in comparison to the original.

Conclusion While many CPRs are developed, they are often not validated externally. Of those that have been validated, it is often difficult to uphold the same level of performance. The majority of externally validated CPRs assessed in this study meet geographical and temporal transportability which is a crucial feature in the CPR's ability to uphold performance amongst different populations.

\section{GP156 INCREASING RATES OF SELF-HARM AMONG CHILDREN 10-14 IN IRELAND: A TEN-YEAR NATIONAL REGISTRY STUDY 2007-2016}

\footnotetext{
${ }^{1,2,3}$ Fiona McNicholas* ${ }^{*}{ }^{4,5}$ Helen Keeley, ${ }^{5,6}$ eve griffin, ${ }^{6}$ Elaine McMahon, ${ }^{6,7}$ Paul Corcoran, 7,6Ivan J Perry, 7,6Ella Arensman. ${ }^{1}$ UCD, Dublin, Ireland; ${ }^{2}$ OLCHC, Dublin, Ireland; ${ }^{3}$ Lucena Clinic SJOG, Dublin, Ireland; ${ }^{4}$ CAMHS HSE South Cork, Cork, Ireland; ${ }^{5}$ UCC, Cork, Ireland; ${ }^{6}$ National Suicide Research Foundation, Cork, Ireland; ' 5 School Public Health UCC, Cork, Ireland
}

\subsection{6/archdischild-2019-epa.220}

Despite stabilization in suicide rates in most countries, rates of Self Harm have continued to increase in young people. Research abounds as to the risks and protective factors but there is still a huge gap in our knowledge as to what leads one person to act on suicidal ideation and another to refrain, and what causes increasing numbers to engage in repetitive non-suicidal self-(NSSI).

The current study examined trends in rates and trends of self-harm among young people in Ireland aged 10-14 years over a ten-year period.

Methods Data from the National Self-Harm Registry Ireland on presentations to hospital emergency departments (EDs) following self-harm in 10-14 years-old during the period 2007-2016 were included. We calculated annual self-harm rates per 100,000 by age, sex and method of self-harm. Poisson Regression models were used to examine trends in rates.

Results The rate of self-harm was 72 per 100,000 (34 for males and 112 for females) an increase of $75 \%$ between 2007 and 2016 (IRR $=1.75,95 \% \mathrm{CI}=1.15-2.10)$, higher in boys $(82 \%$ versus $72 \%)$. Rates of attempted hanging and selfcutting more than doubled ( $I R R=2.43$ and 2.72 , respectively). Attendances to hospital with self-harm increased over the course of the day peaking at $11 \mathrm{pm}$, and and unexpectedly perhaps highest on Mondays.

Presentations involving self-poisoning were highest, and Paracetamol containing medication were involved in the majority of presentations (51\%), 19\% involved NSAIDs and $12 \%$ involved minor tranquilisers (22\% for boys and 9\%) for girls). $8 \%$ of presentations involved alcohol. Considering those discharged from the ED, the majority $(57 \%)$ were referred to 